\title{
Simvastatin in combination with meclofenamic acid inhibits the proliferation and migration of human prostate cancer PC-3 cells via an AKR1C3 mechanism
}

\author{
YOSHITAKA SEKINE, HIROSHI NAKAYAMA, YOSHIYUKI MIYAZAWA, \\ HARUO KATO, YOSUKE FURUYA, SEIJI ARAI, HIDEKAZU KOIKE, \\ HIROSHI MATSUI, YASUHIRO SHIBATA, KAZUTO ITO and KAZUHIRO SUZUKI
}

Department of Urology, Gunma University Graduate School of Medicine, Maebashi, Gunma 371-8511, Japan

Received July 25, 2017; Accepted December 11, 2017

DOI: $10.3892 / \mathrm{ol} .2017 .7721$

\begin{abstract}
Statins have become of interest in research due to their anticancer effects. However, the exact mechanism of their anticancer properties remains unclear. The authors previously reported that statins decrease intracellular cholesterol levels in androgen-independent prostate cancer cells. In de novo androgen synthesis, cholesterol is the primary material and certain enzymes have important roles. The present study aimed to determine whether simvastatin alters the expression of androgen synthesis-associated enzymes in androgen-independent prostate cancer cells. A novel combination therapy of statins and other drugs that inhibit the overexpression of enzymes involved in androgen synthesis was explored. The cytotoxicity of simvastatin and meclofenamic acid was assessed in prostate cancer cells using MTS and migration assays. Testosterone and dihydrotestosterone concentrations in the culture medium were measured using liquid chromatography-tandem mass spectrometry. RAC- $\alpha$-serine/threonine-protein kinase (Akt) phosphorylation was detected by western blot analysis. Following treatment with simvastatin, aldo-keto reductase family 1 member C3 (AKR1C3) expression increased in PC-3 ( $>60$-fold) and LNCaP-LA cells, however not in 22Rv1 cells. Small interfering (si)RNA was used to clarify the effects of AKR1C3 expression. The reduction in AKR1C3 expression
\end{abstract}

Correspondence to: Dr Yoshitaka Sekine, Department of Urology, Gunma University Graduate School of Medicine, 3-9-22 Showa-machi, Maebashi, Gunma 371-8511, Japan

E-mail: ysekine@gunma-u.ac.jp

Abbreviations: AKR1C3, aldo-keto reductase family 1 member C3; IGF1R, insulin-like growth factor 1 receptor; CRPC, castration-resistant prostate cancer, DHT, dihydrotestosterone; LC-MS/MS, liquid chromatography coupled with tandem mass spectrometry; FBS, fetal bovine serum; IGF, insulin-like growth factor; NSAIDs, non-steroidal anti-inflammatory drugs

Key words: prostate cancer, statins, meclofenamic acid, AKR1C3 in PC-3 cells following siRNA transfection was not associated with basal cell proliferation and migration; however, treatment with simvastatin decreased cell proliferation and migration. The combination of simvastatin and meclofenamic acid, an AKR1C3 inhibitor, further enhanced the inhibition of cell proliferation and migration compared with treatment with either drug alone. Furthermore, treatment with simvastatin attenuated insulin-like growth factor 1-induced Akt activation; however, the combination of simvastatin and meclofenamic acid further inhibited Akt activation. These results suggest that the combination of simvastatin and meclofenamic acid may be an effective strategy for the treatment of castration-resistant prostate cancer.

\section{Introduction}

A statin is a drug used to treat hyperlipidemia and functions by inhibiting 3-hydroxy-3-metylglutaryl coenzyme A reductase. Statins have gained much recent attention due to their anticancer effects. Previous studies have shown that statins can prolong survival, while others have reported no benefits in cancer patients (1). Concerning prostate cancer, the anticancer effect of statins is controversial $(2,3)$. We previously reported that statins inhibit prostate cancer progression via suppressing the expression of insulin-like growth factor 1 receptor (IGF1R) and increasing ANXA10 $(4,5)$. However, the exact mechanism of their anticancer properties remains unclear.

There has been recent interest and concerns regarding intratumoral de novo androgen synthesis in castration-resistant prostate cancer (CRPC). Now we are treating CRPC patients with enzalutamide and abiraterone, which attenuate the effects of intratumoral de novo androgens. In de novo androgen synthesis, cholesterol is the primary material, and various enzymes play important roles. We previously reported that intracellular cholesterol levels are decreased in androgen-independent prostate cancer cells after treatment with simvastatin (6); however, alterations in androgen synthesis-related enzymes are not clear.

In this study, we determined whether simvastatin alters the expression of enzymes involved in androgen synthesis in CRPC cells. We also explored a new combination therapy 
of statins and other drugs that inhibit the overexpression of androgen synthesis-related enzymes.

\section{Materials and methods}

Cells and chemicals. Human prostate cancer cell lines PC-3, LNCaP, and 22RV1 were purchased from DS Pharma Biomedical (Osaka, Japan) and cultured in RPMI 1640 (Sigma-Aldrich; Merck KGaA, Darmstadt, Germany) supplemented with 10\% FBS (Moregate BioTech, Bulimba, Australia). PC-3 is an androgen receptor-negative human prostate cancer cell line (7). LNCaP-LA cells, which were generated from LNCaP cells, were cultured in medium containing $10 \%$ charcoal-stripped fetal bovine serum (FBS) for more than 3 months.

Measurement of testosterone and dihydrotestosterone (DHT) in culture medium. Cells were cultured on a 6-well plate and incubated overnight in medium containing 10\% FBS. Cells were then incubated with or without simvastatin $(5 \mu \mathrm{M})$. After $48 \mathrm{~h}$, androstenedione $(100 \mu \mathrm{M})$ was added to the medium. After $24 \mathrm{~h}$, culture medium was collected, and testosterone and DHT concentrations were measured using liquid chromatography-tandem mass spectrometry (LC-MS/MS) (ASKA Pharmaceutical Medical Co., Ltd., Kawasaki, Japan). RIPA buffer was added to wells and protein concentration was measured by the DC Protein Assay (Bio-Rad Laboratories, Inc., Hercules, CA, USA). Testosterone and DHT levels were calculated by dividing the results of the protein assay by the total protein concentration.

$R T-q P C R$. Transcript levels were quantified using the Applied Biosystems 7300 Real-Time PCR system (Applied Biosystems; Thermo Fisher Scientific, Inc., Waltham, MA, USA) according to the manufacturer's protocol. Total RNA was extracted, cDNA was synthesized (8), and polymerase chain reaction (PCR) amplification was performed, using $2 \mu \mathrm{l}$ cDNA and the StAR, CYP11A1, CYP17A1, aldo-keto reductase family 1 member C3 (AKR1C3), HSD3B1, HSD3B2, SRD5A1, SRD5A2, and AKR1C2 primers (No. Hs00986559_ g1, Hs00167984_m1, Hs01124136_m1, Hs00366267_m1, Hs00426435_m1, Hs00605123_m1, Hs00602694_mH, Hs00165843_m1, and Hs00912742_m1, respectively; Applied Biosystems). Next, PCR was performed for one cycle of $10 \mathrm{~min}$ at $95^{\circ} \mathrm{C}$ followed by 40 cycles of $15 \mathrm{sec}$ at $95^{\circ} \mathrm{C}$ and $60 \mathrm{sec}$ at $60^{\circ} \mathrm{C}$. b-Actin (No. $4326315 \mathrm{E}$, Applied Biosystems) transcript levels were used as the internal control. mRNA fold changes were quantified using $\Delta \Delta \mathrm{Cq}$.

MTS assay. Cells were plated onto a 96-well plate in $100 \mu \mathrm{l}$ culture medium containing $10 \%$ FBS. After $24 \mathrm{~h}$, cells were incubated with medium containing simvastatin $(5 \mu \mathrm{M})$ and/or meclofenamic acid $(50 \mu \mathrm{M})$. After incubation for $48 \mathrm{~h}$, the number of living cells was measured using the MTS assay.

Migration assay. Cells were plated onto a 12-well plate and grown to confluence. A $1,000-\mu 1$ tip was used to make a denuded area. Cells were washed twice with PBS and incubated with medium containing various concentrations of simvastatin for $48 \mathrm{~h}$. Mitomycin $\mathrm{C}(0.5 \mu \mathrm{M})$ was added to the medium to inhibit cell proliferation. Photographs were taken at 0 and $48 \mathrm{~h}$, and the distance of cell migration was determined by subtracting the values obtained at $0 \mathrm{~h}$ from those obtained at $48 \mathrm{~h}$. Migration distance is expressed as fold change over the control.

siRNA transfection. Cells were transfected with ON-TARGETplus Non-targeting Pool (no. D-001810-10-05; Dharmacon, Waltham, MA, USA) or ON-TARGETplus AKR1C3 siRNA (No. L-008116-00-0005, Dharmacon) using DharmaFect 2 (Dharmacon). Cells were incubated for $48 \mathrm{~h}$ after transfection.

Western blot analysis. Cell lysates were prepared in RIPA buffer containing $1 \mathrm{mM}$ sodium orthovanadate(Sigma-Aldrich; Merck KGaA) and Halt Protease Inhibitor Cocktail (Pierce; Thermo Fisher Scientific, Inc.). Samples were boiled for 5 min; an equal amount of protein (30 $\mu \mathrm{g} /$ lane) was subjected to $4-12 \%$ SDS-PAGE and transferred onto nitrocellulose membranes. Each membrane was incubated with the following primary polyclonal antibodies: rabbit anti-Akt $(1: 1,000)$, rabbit anti-phospho-Akt (Ser473) $(1: 1,000)$ (Cell Signaling Technology, Inc., Beverly, MA, USA). Blots were developed using a 1:2,000 dilution of the HRP-conjugated secondary antibody (Cell Signaling Technology, Inc.). Proteins were visualized using Immobilon (Merck Millipore, Darmstadt, Germany).

Statistical analysis. Data are expressed as the mean \pm standard deviation. Differences between values were evaluated by one-way ANOVA using Tukey's post hoc analysis and Student's t-test. $\mathrm{P}<0.05$ was considered to indicate a statistically significant difference.

\section{Results}

Simvastatin altered the expression of genes encoding steroidogenic enzymes in androgen-independent prostate cancer cells. We examined PC-3, LNCaP-LA and 22Rv1 cells to determine whether simvastatin alters genes that encode steroidogenic enzymes in androgen-independent prostate cancer cells. After treatment with simvastatin, the expression of AKR1C3 was increased in PC-3 and LNCaP-LA cells (Figs. 1A and 2A) but not in 22Rv1 cells (data not shown). Moreover, the fold change was more than 60 times in PC-3 cells. Conversely, the expression of hydroxy-delta-5-steroid dehydrogenase, 3 beta- and steroid delta-isomerase 1 (HSD3B1) was decreased in PC-3 and LNCaP-LA cells (Figs. 1A and 2A) but not in 22Rv1 cells (data not shown). Moreover, simvastatin increased steroid 5 alpha-reductase 1 (SRD5A1) expression in PC-3 (Fig. 1A) but not in LNCaP-LA or 22Rv1 cells (data not shown). The expression of steroidogenic acute regulatory protein (StAR), cytochrome P450 family 11 subfamily A member 1 (CYP11A1), cytochrome P450 family 17 subfamily A member 1 (CYP17A1), hydroxy-delta-5-steroid dehydrogenase, 3 beta- and steroid delta-isomerase 2 (HSD3B2), steroid 5 alpha-reductase 2 (SRD5A2), and aldo-keto reductase family 1 member C2 (AKR1C2) did not change following treatment with simvastatin (data not shown). 
A

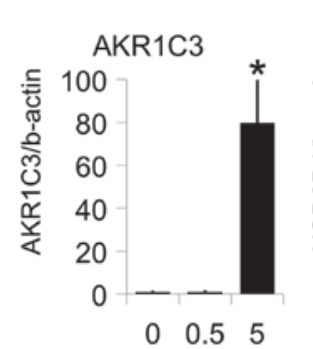

Simvastatin $(\mu \mathrm{M})$

B LNCAP-LA

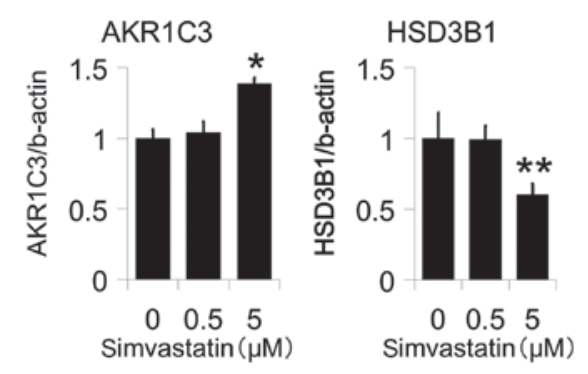

Figure 1. Effects of simvastatin on the expression of genes encoding steroidogenic enzymes in androgen-independent prostate cancer cells (A) PC-3 and (B) LNCaP-LA. Cells were incubated in medium containing various concentrations of simvastatin. After $48 \mathrm{~h}$, we harvested the cells, and the mRNA expression levels of genes encoding steroidogenic enzymes were evaluated by qPCR. The temporal changes of gene expressions were evaluated following simvastatin treatment. Values are expressed as the mean $\pm \mathrm{SD}(\mathrm{n}=4) .{ }^{*} \mathrm{P}<0.01$ vs. $0 \mu \mathrm{M},{ }^{* *} \mathrm{P}<0.05$ vs. $0 \mu \mathrm{M}$.

Effects of AKR1C3 expression on testosterone and DHT levels in PC-3 cell culture medium. To determine whether increased levels of AKR1C3 affect the de novo synthesis of intracellular androgen, we measured the testosterone and DHT levels in culture medium following treatment with simvastatin by LC-MS/MS. Simvastatin significantly increased both testosterone (Fig. 2A) and DHT (Fig. 2B) levels after the addition of androstenedione. These data show that the up-regulation of AKR1C3 is functional.

AKRIC3 inhibition increased the simvastatin-induced inhibition of cell proliferation and migration. PC-3 is an AR-negative human prostate cancer cell line. Therefore, there is a possibility that an increase in testosterone and DHT levels does not affect cell viability. In contrast, the overexpression of AKR1C3 promotes angiogenesis and aggressiveness in PC-3 cells (9). To further determine whether increased AKR1C3 expression affects simvastatin-induced cell viability, AKR1C3 expression was reduced by transfection with siRNA against AKR1C3. siRNA treatment inhibited the expression of AKR1C3 mRNA in $\mathrm{PC}-3$ cells (Fig. 3A). The reduction in AKR1C3 expression in PC-3 cells following siRNA transfection was not associated with basal cell proliferation and migration; however, siRNA transfection with simvastatin significantly decreased both cell proliferation (Fig. 3B) and cell migration (Fig. 3C and D) compared to simvastatin alone.

Meclofenamic acid increased the simvastatin-induced inhibition of cell proliferation and migration. Some drugs are reported to inhibit AKR1C3. Meclofenamic acid is an NSAID
A

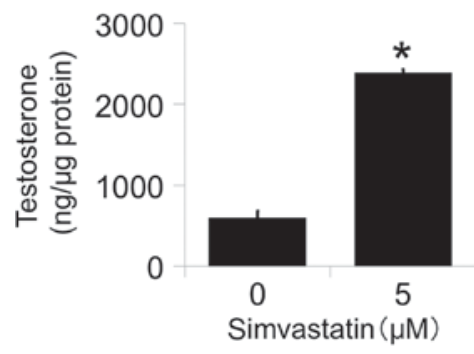

B

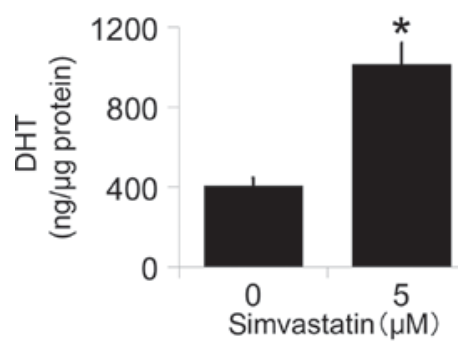

Figure 2. Effects of simvastatin on testosterone and dihydrotestosterone (DHT) levels in PC-3 cell culture medium. (A) Testosterone and (B) DHT concentrations were measured by LC-MS/MS. Values are expressed as the mean $\pm \mathrm{SD}(\mathrm{n}=3) .{ }^{*} \mathrm{P}<0.01$ vs. $0 \mu \mathrm{M}$.

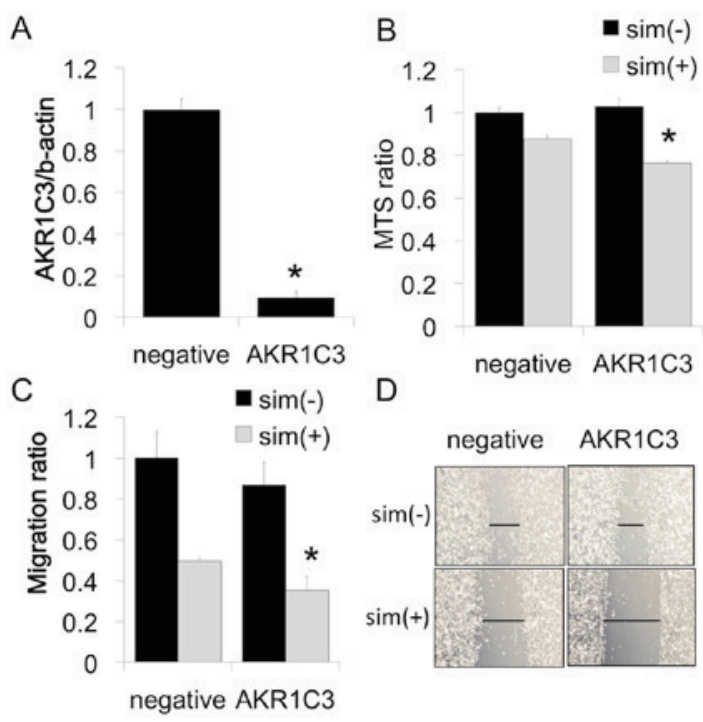

Figure 3. Effects of AKR1C3 siRNA on PC-3 cells. (A) Effects of siRNA on ABCA1 expression in PC-3 cells. Cells transfected with AKR1C3 siRNA (AKR1C3) or non-specific control siRNA (negative). Cells were incubated for $48 \mathrm{~h}$ before harvest for real-time PCR. Values are expressed as the mean $\pm \mathrm{SD}$ $(\mathrm{n}=3)$. ${ }^{*} \mathrm{P}<0.01$ vs. non-specific control siRNA. (B) After transfection of AKR1C3 or non-specific control siRNA, PC-3 cells were incubated with medium containing $10 \%$ FBS. After 48 h, PC-3 cells were cultured with or without simvastatin $(5 \mu \mathrm{M})$ in medium containing $10 \% \mathrm{FBS}$. After $48 \mathrm{~h}$, the number of viable cells was evaluated by the MTS assay. Values are expressed as the mean $\pm \mathrm{SD}(\mathrm{n}=4)$. ${ }^{*} \mathrm{P}<0.01$ vs. negative/sim(+). (C and $\left.\mathrm{D}\right)$ After transfection, PC-3 cells were incubated with medium containing $10 \%$ FBS. After $48 \mathrm{~h}$, cells were wounded and cultured for $48 \mathrm{~h}$ in the presence or absence of simvastatin $(5 \mu \mathrm{M})$. Cell migration into the wound was examined by phase-contrast microscopy. Values are expressed as the mean $\pm \operatorname{SD}(n=3)$. ${ }^{*} \mathrm{P}<0.05$ vs. negative/sim(+).

as well as one of the best inhibitors of AKRs, especially AKR1C3 (10). Therefore, we evaluated the combinatory effects of simvastatin and meclofenamic acid in PC-3 cells. Treatment 
A

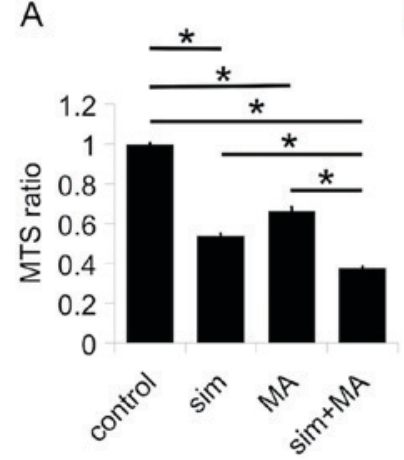

B

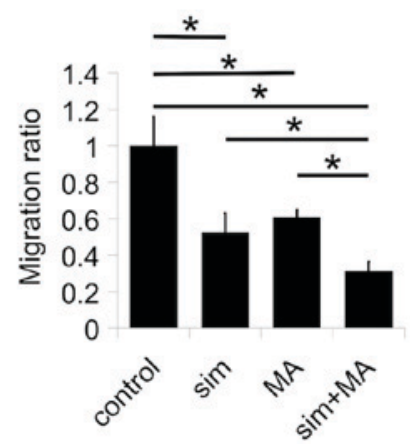

C

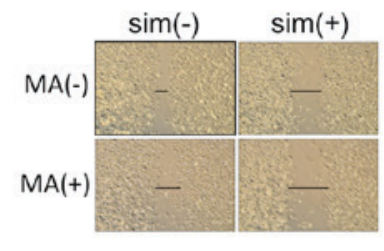

Figure 4. Effects of combination simvastatin with meclofenamic acid on PC-3 cells. (A) PC-3 cells were incubated with medium containing $10 \%$ FBS. After $48 \mathrm{~h}$, the PC-3 cells were cultured with or without simvastatin $(5 \mu \mathrm{M})$ and meclofenamic acid $(50 \mu \mathrm{M})$ in medium containing $10 \%$ FBS. After $48 \mathrm{~h}$, the number of viable cells was evaluated by the MTS assay. Values are expressed as the mean \pm SD ( $n=4)$. * $\mathrm{P}<0.01$. (B and C) PC-3 cells were incubated with medium containing $10 \%$ FBS. After $48 \mathrm{~h}$, cells were wounded and cultured with or without simvastatin $(5 \mu \mathrm{M})$ and meclofenamic acid (50 $\mu \mathrm{M})$. After $48 \mathrm{~h}$, cell migration into the wound was examined by phase-contrast microscopy. Values are expressed as the mean \pm SD $(n=3)$. * $\mathrm{P}<0.01$. sim, simvastatin.

with either simvastatin or meclofenamic acid alone inhibited cell proliferation (Fig. 4A) and migration (Fig. 4B and C). The combination of the two drugs further enhanced cell proliferation (Fig. 4A) and migration (Fig. 4B and C).

Combination of simvastatin and meclofenamic acid inhibited IGF1-induced Akt activation. AKR1C3 overexpression induces Akt activation in PC-3 cells (9). We previously showed that simvastatin without IGF1 decreases IGF1R expression strongly in PC-3 cells (4). IGF1-Akt activation is a well-known pathway in prostate cancer. We hypothesized that inhibiting simvastatin-stimulated AKR1C3 expression with an AKR1C3 inhibitor would have a synergistic effect on simvastatin-blocked IGF1-induced Akt activation. Therefore, the effects of the combination of simvastatin and meclofenamic acid on IGF1-induced Akt activation were evaluated in PC-3 cells. Treatment with either simvastatin or meclofenamic acid alone attenuated IGF1-induced Akt activation, whereas the combination of simvastatin and meclofenamic acid further inhibited Akt activation (Fig. 5).

\section{Discussion}

The main finding of the present study was that simvastatin increased AKR1C3 expression in androgen-independent prostate cancer cells. Furthermore, the combination of simvastatin and meclofenamic acid, an AKR1C3 inhibitor, further suppressed PC-3 cell proliferation, migration, and Akt activation compared with simvastatin alone.

Statins have recently been studied for their pleiotrophic effects, which may make them relevant for cancer prevention or treatment. Clinical reports have shown that statin use is beneficial for overall survival and cancer-specific survival both before and after prostate cancer diagnosis $(1,2)$. In contrast, Platz et al reported that the use of statin drugs was not associated with the overall risk of prostate cancer (3). In vitro, statins exert many biological activities that inhibit prostate cancer progression (e.g., lowering raft cholesterol content, inhibiting cyclin-dependent-kinase-2 activity, decreasing IGF1 receptor expression, and increasing ANXA10 expression) $(4,5,11,12)$. These results indicate that statins have anticancer potential.

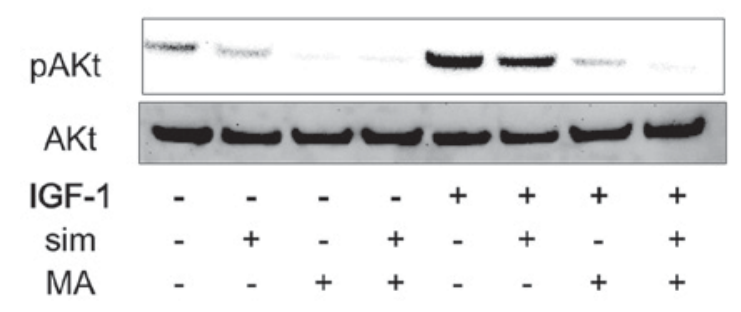

Figure 5. Effects of the combination of simvastatin with meclofenamic acid on IGF-1-induced Akt activation in PC-3 cells. PC-3 cells were incubated with medium containing $10 \%$ FBS. After 48 h, PC-3 cells were cultured with or without simvastatin $(5 \mu \mathrm{M})$ and meclofenamic acid $(50 \mu \mathrm{M})$ in medium containing $1 \%$ FBS. After $48 \mathrm{~h}$, the cells were cultured with or without IGF-1 (40 $\mathrm{ng} / \mathrm{ml}$ ) for $120 \mathrm{~min}$. All cells were harvested at the same time and cell lysates were prepared for western blotting. A representative experiment is shown, which was repeated three times with similar results. sim, simvastatin; MA, meclofenamic acid.

Statins inhibit 3-hydroxy-3-metylglutaryl coenzyme A reductase, one of the most important players in cholesterol biosynthesis. In androgen-dependent prostate cancer cells, statins do not lower intracellular cholesterol levels by up-regulating the low density lipoprotein receptor in the same manner as in normal cells (6). Conversely, statins decrease intracellular cholesterol levels in androgen-independent prostate cancer cells, which cannot regulate low density lipoprotein receptor expression (6). In this study, we focused on the decrease in intracellular cholesterol levels following treatment with statins in CRPC. Recent reports have shown that de novo androgen synthesis is a therapeutic objective in CRPC (13). In the androgen synthesis pathway, cholesterol is the primary material. Therefore, we examined the effects of statins on androgen synthesis-related enzymes in CRPC cells.

Simvastatin increased the expression of AKR1C3 in PC-3 cells. AKR1C3 exhibits $3 \alpha-, 17 \beta$ - and $20 \alpha$-hydroxysteroid dehydrogenase activities (14). The expression of AKR1C3 is increased in several human cancers, including kidney (15) and breast (16). Concerning prostate cancer, localized, metastatic and recurrent prostate cancer has high levels of AKR1C3 (17-19). In addition, elevated AKR1C3 expression promotes the aggressiveness of PC-3 cells, which lack AR (7). These data indicate that increased levels of AKR1C3 induce prostate cancer progression not only by synthesizing 
intracellular androgen but also by androgen-independent mechanisms.

Medical agents such as non-steroidal anti-inflammatory drugs (NSAIDs), steroids, flavonoids, cyclopentane derivatives, and benzodiazepines inhibit AKR1C3 (20). One example of an NSAID is meclofenamic acid, which inhibits AKR1C3, cyclooxygenase-1, and cyclooxygenase-2 more strongly than other NSAIDs $(21,22)$. Meclofenamic acid inhibits androgen-independent prostate cancer progression both in vitro and in vivo (23). In this study, AKR1C3 siRNA did not affect PC-3 cell proliferation and migration, whereas meclofenamic acid inhibited these processes, suggesting that meclofenamic acid also has anticancer effects without the AKR1C3 mechanism. The combination of simvastatin and meclofenamic acid inhibited PC-3 cell proliferation, migration, and Akt activation to a greater extent than simvastatin or meclofenamic acid alone. Previous reports have described combination therapy using statins and NSAIDs for the treatment of prostate cancer, which works by inhibiting NF- $\mathrm{BB}$ (24) or IL-6 (25). These results reveal that the inhibition of AKR1C3 is an underlying mechanism of the combination therapy of simvastatin and meclofenamic acid.

The present study had several limitations. First, three androgen-independent prostate cancer cell lines responded to simvastatin with different levels of AKR1C3 expression. Prostate cancer is very heterogenic. In particular, androgen-independent prostate cancer cells have a different genetic background (26). Therefore, our results may not be applicable to all CRPCs. In addition, we evaluated the effects of simvastatin and meclofenamic acid only in PC-3 cells, and studied the changes of gene expressions following simvastatin treatment only in one single time rather than performing a time-course experiment. Concerning Akt activation by IGF-1, we also checked only in one single time and IGF1R phosphorylation status was not evaluated. Moreover, in vivo models are required to show whether the combination of simvastatin and meclofenamic acid may have a curative influence on CRPC.

In summary, simvastatin increased AKR1C3 expression in androgen-independent prostate cancer cells, and the combination of simvastatin and meclofenamic acid further inhibited PC-3 cell proliferation, migration and Akt activation compared with simvastatin alone. These results suggest that the combination of statin and NSAIDs may be an effective strategy for the treatment of prostate cancer.

\section{Acknowledgements}

We thank Ms. Naomi Takase, Ms. Atsuko Oyama, and Ms. Hayumi Oyama for their technical assistance. This work was supported by JSPS KAKENHI Grant no. 25861410.

\section{References}

1. Zhong S, Zhang X, Chen L, Ma T, Tang J and Zhao J: Statin use and mortality in cancer patients: Systematic review and meta-analysis of observational studies. Cancer Treat Rev 41: 554-567, 2015.

2. Bansal D, Undela K, D'Cruz S and Schifano F: Statin use and risk of prostate cancer: A meta-analysis of observational studies. PLoS One 7: e46691, 2012
3. Platz EA, Tangen CM, Goodman PJ, Till C, Parnes HL, Figg WD, Albanes D, Neuhouser ML, Klein EA, Lucia MS, et al: Statin drug use is not associated with prostate cancer risk in men who are regularly screened. J Urol 192: 379-384, 2014.

4. Sekine Y, Furuya Y, Nishii M, Koike H, Matsui H and Suzuki K: Simvastatin inhibits the proliferation of human prostate cancer PC-3 cells via down-regulation of the insulin-like growth factor 1 receptor. Biochem Biophys Res Commun 372: 356-361, 2008.

5. Miyazawa Y, Sekine Y, Kato H, Furuya Y, Koike H and Suzuki K: Simvastatin Up-regulates annexin A10 that can inhibit the proliferation, migration, and invasion in androgen-independent human prostate cancer cells. Prostate 77: 337-349, 2017.

6. Furuya Y, Sekine Y, Kato H, Miyazawa Y, Koike H and Suzuki K: Low-density lipoprotein receptors play an important role in the inhibition of prostate cancer cell proliferation by statins. Prostate Int 4: 56-60, 2016.

7. Sobel RE and Sadar MD: Cell lines used in prostate cancer research: A compendium of old and new lines-part 1. J Urol 173: 342-359, 2005.

8. Suzuki K, Koike H, Matsui H, Ono Y, Hasumi M, Nakazato H, Okugi H, Sekine Y, Oki K, Ito K, et al: Genistein, a soy isoflavone, induces glutathione peroxidase in the human prostate cancer cell lines LNCaP and PC-3. Int J Cancer 99: 846-852, 2002.

9. Dozmorov MG, Azzarello JT, Wren JD, Fung KM, Yang Q, Davis JS, Hurst RE, Culkin DJ, Penning TM and Lin HK: Elevated AKR1C3 expression promotes prostate cancer cell survival and prostate cell-mediated endothelial cell tube formation: Implications for prostate cancer progression. BMC Cancer 10: 672, 2010.

10. Flanagan JU, Yosaatmadja Y, Teague RM, Chai MZ, Turnbull AP and Squire CJ: Crystal structures of three classes of non-steroidal anti-inflammatory drugs in complex with aldo-keto reductase 1C3. PLoS One 8: e43965, 2012.

11. Zhuang L, Kim J, Adam RM, Solomon KR and Freeman MR: Cholesterol targeting alters lipid raft composition and cell survival in prostate cancer cells and xenografts. J Clin Invest 115: 959-968, 2005.

12. Sivaprasad U, Abbas Tand Dutta V: Differential efficacy of 3-hydroxy-3-methlyglutaryl CoA reductase inhibitors on the cell cycle of prostate cancer cells. Mol Cancer Ther 5: 2310-2316, 2006.

13. Cai C, Chen S, Ng P, Bubley GJ, Nelson PS, Mostaghel EA, Marck B, Matsumoto AM, Simon NI, Wang H, et al: Intratumoral de novo steroid synthesis activates androgen receptor in castration-resistant prostate cancer and is upregulated by treatment with CYP17A1 inhibitors. Cancer Res 71: 6503-6513, 2011

14. Matsuura K, Shiraishi H, Hara A, Sato K, Deyashiki Y, Ninomiya $M$ and Sakai S: Identification of a principal mRNA species for human 3alpha-hydroxysteroid dehydrogenase isoform (AKR1C3) that exhibits high prostaglandin D2 11-ketoreductase activity. J Biochem 124: 940-946, 1998.

15. Azzarello JT, Lin HK, Gherezghiher A, Zakharov V, Yu Z, Kropp BP, Culkin DJ, Penning TM and Fung KM: Expression of AKR1C3 in renal cell carcinoma, papillary urothelial carcinoma, and Wilms' tumor. Int J Clin Exp Pathol 3: 147-155, 2009.

16. Byrns MC, Duan L, Lee SH, Blair IA and Penning TM: Aldo-keto reductase 1C3 expression in MCF-7 cells reveals roles in steroid hormone and prostaglandin metabolism that may explain its over-expression in breast cancer. J Steroid Biochem Mol Biol 118: 177-187, 2010.

17. Nakamura Y, Suzuki T, Nakabayashi M, Endoh M, Sakamoto K, Mikami Y, Moriya T, Ito A, Takahashi S, Yamada S, et al: In situ androgen producing enzymes in human prostate cancer. Endocr Relat Cancer 12: 101-107, 2005.

18. Stanbrough M, Bubley G, Ross K, Golub TR, Rubin MA, Penning TM, Febbo PG and Balk SP: Increased expression of genes converting adrenal androgens to testosterone in androgen-independent prostate cancer. Cancer Res 66: 2815-2825, 2006.

19. Wako K, Kawasaki T, Yamana K, Suzuki K, Jiang S, Umezu H, Nishiyama T, Takahashi K, Hamakubo T, Kodama T and Naito M: Expression of androgen receptor through androgen-converting enzymes is associated with biological aggressiveness in prostate cancer. J Clin Pathol 61: 448-454, 2008.

20. Byrns MC, Jin Y and Penning TM: Inhibitors of type 5 17 $\beta$-hydroxysteroid dehydrogenase (AKR1C3): Overview and structural insights. J Steroid Biochem Mol Biol 125: 95-104, 2011. 
21. Kalgutkar AS, Crews BC, Rowlinson SW, Marnett AB Kozak KR, Remmel RP and Marnett LJ: Biochemically based design of cyclooxygenase-2 (COX-2) inhibitors: Facile conversion of nonsteroidal antiinflammatory drugs to potent and highly selective COX-2 inhibitors. Proc Natl Acad Sci USA 97: 925-930, 2000.

22. Ouellet M and Percival MD: Effect of inhibitor time-dependency on selectivity towards cyclooxygenase isoforms. Biochem J 306: $247-251,1995$.

23. Soriano-Hernández AD, Galvan-Salazar HR, Montes-Galindo DA, Rodriguez-Hernandez A, Martinez-Martinez R, Guzman-Esquivel J, Valdez-Velazquez LL, Baltazar-Rodriguez LM, Espinoza-Gómez F, Rojas-Martinez A, et al: Antitumor effect of meclofenamic acid on human androgen-independent prostate cancer: A preclinical evaluation. Int Urol Nephrol 44: 471-477. 2012.
24. Zheng X, Cui XX, Avila GE, Huang MT, Liu Y, Patel J, Kong AN, Paulino R, Shih WJ, Lin Y, et al: Atorvastatin and celecoxib inhibit prostate PC-3 tumors in immunodeficient mice. Clin Cancer Res 13: 5480-5487, 2007.

25. Wang H, Cui XX, Goodin S, Ding N, Van Doren J DuZ, Huang MT, Liu Y, Cheng X, Dipaola RS, et al: Inhibition of IL-6 expression in $\mathrm{LNCaP}$ prostate cancer cells by a combination of atorvastatin and celecoxib. Oncol Rep 31: 835-841, 2014.

26. Fraser M, Berlin A, Bristow RG and van der Kwast T: Genomic, pathological, and clinical heterogeneity as drivers of personalized medicine in prostate cancer. Urol Oncol 33: 85-94, 2015. 